\title{
Decomposability of Projective Curvature Tensor in Recurrent Finsler Space $\left(W R-F_{n}\right)$
}

\author{
Meenakshy Thakur \\ Department of Mathematics \\ and Statistics \\ Dr. R.M.L. Avadh University, \\ Uttar Pradesh, India
}

\author{
C. K. Mishra \\ Department of Mathematics \\ and Statistics \\ Dr. R.M.L. Avadh University, \\ Uttar Pradesh, India
}

\author{
Gautam Lodhi \\ Department of Mathematics, \\ BBD University, Lucknow \\ Uttar Pradesh, India
}

\begin{abstract}
The decomposition of curvature tensor field was studied by K. Takano[1]. The decomposability of curvature tensor in Finsler manifold was studied by Pandey[2]. The purpose of the present chapter is to decompose the Projective curvature tensor in recurrent Finsler space and study the properties of conformal decomposition tensor.
\end{abstract}

\section{Keywords}

Finsler space, projective curvature tensor, recurrent Finsler space.

\section{INTRODUCTION}

We considered an n-dimensional Finsler space $F_{n}$ in which the projective curvature tensor, projective tensor field and deviation tensor field are defined by Rund[3]

$$
\begin{aligned}
& \text { (a) } W_{j k h}^{i}=H_{j k h}^{i}+\frac{2 \delta_{j}^{i}}{n+1} H_{[j k]}+\frac{2 \dot{x}^{i}}{n+1} \dot{\partial}_{j} H_{[k h]} \\
& +\frac{\delta_{k}^{i}}{n^{2}-1}\left(n H_{j h}+H_{h}+\dot{x}^{r} \dot{\partial}_{j} H_{h r}\right)- \\
& \frac{\delta_{h}^{i}}{n^{2}-1}\left(n H_{j k}+H_{k j}+\dot{x}^{r} \dot{\partial}_{j} H_{k r}\right) \\
& \text { (b) } W_{j k}^{i}=H_{j k}^{i}+\frac{\dot{x}^{i}}{n+1} H_{[j k]} \\
& +2\left\{\frac{\delta_{[j}^{i}}{n^{2}-1}\left(n H_{k]}-\dot{x}^{r} H_{k] r}\right)\right\} \\
& W_{j}^{i}=H_{j k}^{i}-H \delta_{j}^{i}-\frac{1}{n+1}\left(\dot{\partial}_{r} H_{j}^{r}-\dot{\partial}_{j} H\right) \dot{x}^{i},
\end{aligned}
$$

respectively.

The following relations which will be used in our discussion follow from (1.1)(a) and (1.2)(b)

$$
\left\{\begin{array}{l}
\text { a) } W_{j k h}^{i} \dot{x}^{j}=W_{k h}^{i}, \\
\text { b) } W_{k h}^{i} \dot{x}^{k}=W_{h}^{i}, \\
\text { c) } W_{h}^{i} \dot{x}^{h}=0 .
\end{array}\right.
$$

The deviation tensor $W_{k}^{i}$ is homogeneous of second degree in its directional arguments. The Projective tensor $W_{j k}^{i}$ is skewsymmetric in its lower indices and projectively homogeneous of degree one in their directional arguments and the projective curvature tensor $W_{j k h}^{i}$ is skew-symmetric in its indices $k$ and $h$ and is positively homogeneous of degree zero in its directional arguments.
Sinha and Singh[4] have defined that an $F_{n}$ is called Projective recurrent of the first order if the Berwald's covariant derivative of the projective curvature tensor satisfies

$$
W_{j k h(l)}^{i}=V_{l} W_{j k h}^{i},
$$

Where $V_{l}$ is a recurrent vector field. The space equipped with such recurrent vector field and projective curvature tensor is called recurrent Finsler space.

Transvecting (1.4) successively by $\dot{x}^{j}$ and $\dot{x}^{k}$ and therefore using (1.3)(a) and (1.3)(b), we get

$$
\begin{aligned}
& W_{k h(l)}^{i}=V_{l} W_{k h}^{i}, \\
& W_{h(l)}^{i}=V_{l} W_{h}^{i},
\end{aligned}
$$

In view of (1.5) and (1.6). We observe that projective deviation tensor $W_{h}^{i}$ and the Projective tensor $W_{k h}^{i}$ are recurrent.

The projective curvature tensor satisfied the identity by Kumar, Shukla and Tripathi[7]:

$$
W_{h j k(l)}^{i}+W_{h k l(j)}^{i}+W_{h l j(k)}^{i}=0 .
$$

Sinha and Singh [5] have also defined that an $F_{n}$ is called projective recurrent of second order, If the Weyl's projective curvature tensor satisfies

$$
W_{j k h(l)(m)}^{i}=U_{l m} W_{j k h}^{i}
$$

where $U_{l m}$ is a recurrence tensor. Transvecting (1.8) successively by $\dot{x}^{j}$ and $\dot{x}^{k}$, we get

$$
W_{k h(l)(m)}^{i}=U_{l m} W_{k h}^{i}
$$

and

$$
W_{h(l)(m)}^{i}=U_{l m} W_{h}^{i}
$$

Accordingly, we can state that Projective deviation tensor and projective tensor satisfies the second order recurrent condition, if so is Weyl's curvature tensor. 
The recurrent curvature tensor $H_{j k h}^{i}$ satisfies the relation Sinha and Singh[6]:

$$
H_{j k h(l)}^{i}=V_{l} H_{j k h}^{i}
$$

Where $V_{l}$ is recurrence vector. Transvecting (1.12) successively by $\dot{x}^{j}$ and $\dot{x}^{k}$ and, we get

(1.12) $H_{k h(l)}^{i}=V_{l} H_{k h}^{i}$

and

(1.13) $H_{h(l)}^{i}=V_{l} H_{h}^{i}$,

The curvature tensor field of second order satisfies the relations Sinha and Singh[6]:

$$
H_{j k h(l)(m)}^{i}=K_{l m} H_{j k h}^{i},
$$

where $\quad K_{l m}=V_{m}+V_{l(m)}$ is the recurrence tensor. Transvecting successively by $\dot{x}^{j}$ and $\dot{x}^{k}$, we have

$$
H_{k h(l)(m)}^{i}=K_{l m} H_{k h}^{i}
$$

and

(1.16) $H_{h(l)(m)}^{i}=K_{l m} H_{h}^{i}$

\section{DECOMPOSITION OF PROJECTIVE CURVATURE TENSOR IN RECURRENT FINSLER SPACE $\left(W R-F_{n}\right)$}

Let us consider the projective curvature tensor $W_{j k h}^{i}$ in the form

$$
W_{j k h}^{i}=Y_{j}^{i} B_{k h}
$$

where $Y_{j}^{i}$ is non zero tensor and $B_{k h}$ is skew symmetric decomposition tensor.

The space equipped with such decomposition of projective curvature tensor with recurrent Finsler space is called decomposition of Projective curvature tensor in recurrent Finsler space and we denote it by $W R-F_{n}$.

Differentiating (2.1) covariantly with respect to $x^{l}$ in the sense of Berwald's, we get

$$
W_{j k h(l)}^{i}=Y_{J(l)}^{i} B_{k h}+B_{k h(l)} Y_{J}^{i}
$$

Using the equation (1.4), (2.1) in (2.2), we get

$$
W_{j k h(l)}^{i}=\beta_{l} Y_{J}^{i} B_{k h}+B_{k h(l)} Y_{J}^{i}
$$

Where

$$
Y_{J(l)}^{i}=\beta_{l} Y_{J}^{i}
$$

From equation (2.1) and equation (2.3), we get

$$
B_{k h(l)}=\left(V_{l}-\beta_{l}\right) B_{k h} .
$$

Let us assume that $\left(V_{l} \neq \beta_{l}\right)$ then the equation (2.5) may be written as

$$
B_{k h(l)}=\gamma_{l} B_{k h}
$$

where $\gamma_{l}=\left(V_{l}-\beta_{l}\right)$.

Conversely,

If the above equation (2.6) is true then (2.3) yield

$$
V_{l} B_{k h}=\left(\beta_{l}+\gamma_{l}\right) B_{k h}
$$

Accordingly, we have the

Theorem 2.1: In $W R-F_{n}$, the necessary and sufficient condition for the decomposition tensor $B_{k h}$ to be recurrent is that the recurrent vector $V_{l}$ is not equal to recurrent vector $\beta_{l}$.

Let us assume that the vector $V_{l}$ is equal to recurrent vector $\beta_{l}$ such that

(2.8) $\quad V_{l}=\beta_{l}$

In view of equation (2.8), equation (2.6) immediately reduces to

$$
B_{k h(l)}=0 .
$$

Using equation (2.9) in (2.3), we have

$$
\begin{aligned}
W_{j k h(l)}^{i} & =Y_{J(l)}^{i} B_{k h} \\
\text { or } \quad W_{j k h(l)}^{i} & =\beta_{l} Y_{J}^{i} B_{k h}
\end{aligned}
$$

Adding the expressions obtained by cyclic change of (2.10) with respect to the indices $k$, $h$ and $l$, we have

$$
\begin{aligned}
& W_{j k h(l)}^{i}+W_{j h l(k)}^{i}+W_{j l k(h)}^{i} \\
& =Y_{j}^{i}\left(\beta_{l} B_{k h}+\beta_{k} B_{h l}+\beta_{h} B_{l k}\right) .
\end{aligned}
$$

In view of (1.4) equation (2.11) reduces to

$$
Y_{j}^{i}\left(\beta_{l} B_{k h}+\beta_{k} B_{h l}+\beta_{h} B_{l k}\right)=0 .
$$

Since $Y_{j}^{i}$ is non zero tensor, it implies

or

$$
\begin{aligned}
& \beta_{l} B_{k h}+\beta_{k} B_{h l}+\beta_{h} B_{l k}=0 \\
& V_{l} B_{k h}+V_{k} B_{h l}+V_{h} B_{l k}=0
\end{aligned}
$$

Accordingly state:

Theorem 2.2: In $W R-F_{n}$, under the decomposition (2.1), if the vector $V_{l}$ is equal to $\beta_{l}$, the decomposition tensor satisfies the following identity (2.13).

Differentiating (2.10) covariantly with respect to $x^{m}$ in the sense of Berwald's and using (2.9), we get 
(2.14) $W_{j k h(l)(m)}^{i}=\beta_{l(m)} Y_{J}^{i} B_{k h}+\beta_{l} Y_{J(m)}^{i} B_{k h}$

In view of (2.4) the above equation may be written as

$$
W_{j k h(l)(m)}^{i}=\left(\beta_{l(m)}+\beta_{l} \beta_{m}\right) Y_{J}^{i} B_{k h}
$$

Using equation (1.10) and (2.1), we get

$$
U_{l m} Y_{J}^{i} B_{k h}=\left(\beta_{l(m)}+\beta_{l} \beta_{m}\right) Y_{J}^{i} B_{k h}
$$

From (2.15) and (2.16), we have

$$
U_{l m}=\left(\beta_{l(m)}+\beta_{l} \beta_{m}\right)
$$

Thus we conclude that

Theorem 2.3 In $W R-F_{n}$, under the decomposition (2.1), if the vector $V_{l}$ is equal to $\beta_{l}$ for which recurrence vector field $\beta_{l}$ satisfies the condition $\beta_{l(m)}+\beta_{l} \beta_{m} \neq 0$.

Interchanging the indices $l$ and $m$ in (2.15) and subtracting the equation thus obtained to (2.15), we have

$$
\begin{aligned}
& W_{j k h(l)(m)}^{i}-W_{j k h(m)(l)}^{i} \\
& \quad=\left(U_{l m}-U_{m l}\right) Y_{J}^{i} B_{k h} B_{k h} .
\end{aligned}
$$

or

$$
W_{j k h(l)(m)}^{i}-W_{j k h(m)(l)}^{i}=\left(\beta_{l(m)}-\beta_{m(l)}\right) Y_{J}^{i} B_{k h}
$$

Accordingly we state:

Corollary 2.1: In $W R-F_{n}$, Under the decomposition (2.1) if the vector $V_{l}$ is equal to $\beta_{l}$, the projective curvature tensor satisfies the following identity (2.18).

Differentiating (2.6) covariantly with respect to $x^{m}$ in the sense of Berwald's, we get

$$
\begin{aligned}
B_{k h(l)(m)} & =\gamma_{l(m)} B_{k h}+\gamma_{l} B_{k h(m)} \\
& =\left(V_{l(m)}-\beta_{l(m)}\right) B_{k h}+\left(V_{l}-\beta_{l}\right) B_{k h(m)}
\end{aligned}
$$

In view of (2.6), the equation (2.19) may be written as

$$
B_{k h(l)(m)}=\left(V_{l(m)}-\beta_{l(m)}\right) B_{k h}+\gamma_{l}\left(V_{l}-\beta_{l}\right) B_{k h}
$$

or

$$
\begin{array}{r}
B_{k h(l)(m)}=\left(V_{l(m)}-\beta_{l(m)}\right) B_{k h}+ \\
\left(V_{m}-\beta_{m}\right)\left(V_{l}-\beta_{l}\right) B_{k h} \\
B_{k h(l)(m)}=\left(V_{l(m)}-\beta_{l(m)}+V_{m} V_{l}\right. \\
\left.-V_{m} \beta_{l}-V_{l} \beta_{m}+\beta_{l} \beta_{m}\right) B_{k h}
\end{array}
$$

Theorem 2.4:In $W R-F_{n}$, Under the decomposition (2.1), the second order covariant derivative of decomposition tensor $B_{k h}$ satisfies the relation (2.22).

In view of equation (2.8), equation (2.22) immediately reduces to

$$
B_{k h(l)(m)}=0
$$

Corollary 2.2: $W R-F_{n}$, the second order covariant derivative of decomposition tensor $B_{k h}$ vanish, If the vector $V_{l}$ is equal to $\beta_{l}$.

\section{REFERENCES}

[1] K. Takano, 1961, Affine motion in non RiemanninK*space I, \& II, III(with M. Okumura), IV \& V, Tensor, N. S., 11, 137-143, 161-173, 174-181, 245-253, 270-278.

[2] P. N. Pandey, 1981, On decomposability of curvature tensor of a Finsler manifold, ActaMathematica, Academia Scieniarum Hungaricae, Tomus 38(1-4) (1981), 109-116.

[3] H. Rund, 1959, The Differential Geometry of Finsler spaces. Springer-Verlag, Berlin.

[4] B. B. Sinha, S. P. Singh, 1971,On Recurrent Finsler spaces. Rev. Roum. Pures Et Appl Tome XVI, No. 6, Bucarest, 977-986.

[5] B. B. Sinha, S. P. Singh, 1970, On recurrent spaces of second order in Finsler spaces, Yokohama Math. J. 18, 27-32.

[6] B. B. Sinha, S. P. Singh, 1973, Recurrent Finsler Space of second order II, Rep. Indian J. Of pure and App. Math, Vol. 4, No. 1, 45-50.

[7] B. B. Sinha, S. P. Singh and R. P.Tripathi,197, On Recurrent Finsler spaces. Rev. Roum. Pures Et Appl Tome XVI, No. 6 Bucarest, 1971, 977-986. 